\title{
DNA methylation of GHSR, GNG4,HOXD9 and SALL3 is a common epigenetic alteration in thymic carcinoma
}

\author{
REINA KISHIBUCHI ${ }^{1}$, KAZUYA KONDO ${ }^{1}$, SHIHO SOEJIMA ${ }^{1}$, MITSUHIRO TSUBOI ${ }^{2}$, \\ KOICHIRO KAJIURA ${ }^{2}$, YUKIKIYO KAWAKAMI ${ }^{2}$, NAOYA KAWAKITA ${ }^{2}$, TORU SAWADA ${ }^{2}$, \\ HIROAKI TOBA $^{2}$, MITSUTERU YOSHIDA ${ }^{2}$, HIROMITSU TAKIZAWA ${ }^{2}$ and AKIRA TANGOKU ${ }^{2}$ \\ ${ }^{1}$ Department of Oncological Medical Services, Graduate School of Biomedical Sciences, \\ Tokushima University, Tokushima 770-8509; ${ }^{2}$ Department of Thoracic, Endocrine Surgery and Oncology, \\ Graduate School of Biomedical Sciences, Tokushima University, Tokushima 770-8503, Japan
}

Received August 17, 2019; Accepted October 25, 2019

DOI: $10.3892 /$ ijo.2019.4915

\begin{abstract}
Thymic epithelial tumors comprise thymoma, thymic carcinoma and neuroendocrine tumors of the thymus. Recent studies have revealed that the incidence of somatic non-synonymous mutations is significantly higher in thymic carcinoma than in thymoma. However, limited information is currently available on epigenetic alterations in these types of cancer. In this study, we thus performed genome-wide screening of aberrantly methylated $\mathrm{CpG}$ islands in thymoma and thymic carcinoma using Illumina HumanMethylation450 K BeadChip. We identified $92 \mathrm{CpG}$ islands significantly hypermethylated in thymic carcinoma in relation to thymoma and selected $G$ protein subunit gamma 4 (GNG4), growth hormone secretagogue receptor (GHSR), homeobox D9 (HOXD9) and spalt like transcription factor 3 (SALL3), which are related to cancer. We examined the promoter methylation of 4 genes in 46 thymic epithelial tumors and 20 paired thymus tissues using bisulfite pyrosequencing. Promoter methylation was significantly higher in thymic carcinoma than in thymoma and revealed a high discrimination between thymic carcinoma and thymoma in all 4 genes. Promoter methylation was higher in thymic carcinoma than in the thymus. No significant differences were observed in the promoter methylation of $G N G 4$, HOXD 9, or SALL3 between thymoma and the thymus. The
\end{abstract}

Correspondence to: Professor Kazuya Kondo, Department of Oncological Medical Services, Graduate School of Biomedical Sciences, Tokushima University, 3-18-15 Kuramotocho, Tokushima 770-8509, Japan

E-mail:kzykondo@tokushima-u.ac.jp

Abbreviations: TET, thymic epithelial tumor; TC, thymic carcinoma; WHO, World Health Organization; CGI, CpG island; NECTT, neuroendocrine tumor of the thymus; ROC curve, receiver operating characteristic curve

Key words: thymic carcinoma, thymoma, DNA methylation, GNG4, GHSR, HOXD9, SALL3 promoter methylation of the 4 genes was not significantly higher in advanced-stage tumors than in early-stage tumors in all thymic epithelial tumors. Among the 4 genes, relapse-free survival was significantly worse in tumors with a higher DNA methylation than in those with a lower DNA methylation in all thymic epithelial tumors. Moreover, relapse-free survival was significantly worse in thymomas with a higher DNA methylation of HOXD9 and SALL3 than in those with a lower DNA methylation. On the whole, the findings of this study indicated that the promoter methylation of cancer-related genes was significantly higher in thymic carcinoma than in thymoma and the thymus. This is a common epigenetic alteration of high diagnostic value in thymic carcinoma and may be involved in the carcinogenesis of thymic carcinoma. However, epigenetic alterations in the 3 genes, apart from GHSR, are not involved in the tumorigenesis of thymoma.

\section{Introduction}

Although thymic epithelial tumors (TETs) are rare, they are the most common tumor of the anterior mediastinum (1). The World Health Organization (WHO) Consensus Committee (2015) proposed that TETs consist of thymoma (types A, AB, B1, B2 and B3), thymic carcinoma (TC) and neuroendocrine tumors of the thymus (NECTT) (2). Thymoma is defined as a low-grade malignant tumor of the thymic epithelium with a variable population of immature, but non-neoplastic T cells that is associated with myasthenia gravis and other autoimmune diseases. However, TC is defined as a malignant tumor with evidently atypical cells of an invasive nature without immature T-cell infiltration and autoimmune disease (3).

Previous studies have examined genetic alterations in TETs (4-6). Wang et al performed a comparative sequence analysis on $47 \mathrm{TC}$ and 31 thymomas and revealed that the incidence of somatic non-synonymous mutations was significantly higher in TC (62\%) than in thymoma (13\%). They also detected the enrichment of mutations in TP53, BAP1, SETD2, $C Y L D$ and $K I T$ (26-9\%) in TC (4). Radovich et al reported that GTF2IL424H mutations were unique and the most common in type $\mathrm{A}$ and $\mathrm{AB}$ thymomas (type A, 100\%; type AB, 70\%), but rare in other TETs (5). 
Limited information is currently available on epigenetic alterations in TETs $(7,8)$. We previously examined the aberrant DNA methylation of 4 cancer-related genes [the death-associated protein kinase (DAPK), p16, O-6-methylguanine-DNA methyltransferase (MGMT) and hyperpigmentation, progressive, 1 (HPPl) genes] in 26 thymomas and $6 \mathrm{TCs}$ and demonstrated that aberrant methylation was significantly more frequent in TC (86\%) than in thymoma (29\%) (7). We also investigated the DNA methylation of the $M G M T$ gene in 44 thymomas and $23 \mathrm{TCs}$, and found that MGMT methylation was significantly more frequent in TCs (74\%) than in thymomas (29\%). A correlation has been reported between MGMT methylation and the loss of its protein expression (8).

To clarify whether the DNA methylation of certain genes is related to malignant behavior in TET, we herein performed the systematic and genome-wide screening of aberrantly methylated $\mathrm{CpG}$ islands (CGI) in thymoma and TC.

\section{Materials and methods}

Patients and tissue samples. Forty-six TET samples and 20 paired thymic tissues were obtained from patients with histologically proven TET who underwent surgery at Tokushima University Hospital (Tokushima, Japan) between 1990 and 2016. Thymic tissues that were located far from the tumor were obtained during surgery. The patient characteristics are presented in Tables I and SI. All TETs were classified according to the WHO histological classification system (2). The representative pathology of TETs (type A, B1, B2, and B3 thymomas and TC) is illustrated in Fig. S1. The breakdown of TET samples by diagnosis was as follows: 30 cases of thymoma, 12 TC and 4 NECTT. The clinical stage of each TET was identified according to the criteria of Masaoka-Koga staging (9). The frequencies of advanced cases (stages III and IV) of thymoma and TC + NECTT were 33 and $50 \%$, respectively. No significant differences were observed in their frequencies between both groups (Table I, chi-squared test).

The present study was performed in accordance with the principles outlined in the Declaration of Helsinki. Following the approval of all aspects of this study by the local Ethics Committee (Tokushima University Hospital, approval numbers 2205-4), formal written consent was obtained from all patients.

DNA preparation and bisulfite conversion of genomic DNA Tumors were snap-frozen and stored at $-80^{\circ} \mathrm{C}$ until DNA analyses. DNA was extracted using standard methods. The bisulfite conversion of DNA was conducted using the EZ DNA Methylation Gold kit (Zymo Research).

Global methylation analysis. A HumanMethylation450 K BeadChip (Illumina) analysis was performed according to the manufacturer's instructions. The default settings of the GenomeStudio software DNA methylation module (Illumina) were applied to calculate the methylation levels of $\mathrm{CpG}$ sites as $\beta$-values ( $\beta$-intensity methylated/intensity methylated + unmethylated). Data were further normalized using the peak correction algorithm embedded in the Illumina Methylation Analyzer (IMA) R package (10). To identify CGI differentially methylated in B3 type thymoma and TC samples in the discovery set, median-averaged $\beta$-differences in CGI-based regions were calculated based on a matrix of $\beta$-differences, in which the $\beta$-values of TC samples were subtracted from those of B3 thymoma samples. The characteristics of B3 thymomas and TCs in patients are presented in Table SI. The significance of the differences was evaluated using Welch's t-test in IMA. Multiple testing corrections were performed using the Benjamini-Hochberg approach, with significantly differential methylation being defined as a false discovery rate (FDR)-adjusted P-value $<0.05$. The following criteria were used for differentially methylated CGI: $\beta$-difference $>0.5$ and FDR-adjusted P-value $<0.05$. Significant methylated $\mathrm{CpG}$ sites were selected by the Bonferroni's test. Methylation data for the discovery cohort were deposited in the Gene Ontology Database under accession number GSE94769.

Bisulfite pyrosequencing. Bisulfite-treated genomic DNA was amplified using a set of primers designed with PyroMark Assay Design software (version 2.0.01.15; Qiagen; Table SII). PCR product pyrosequencing and methylation quantification were performed with a PyroMark 24 Pyrosequencing System, version 2.0.6 (Qiagen) with sequencing primers designed according to the manufacturer's instructions (Table SII).

Statistical analysis. The Shapiro-Wilk test was used to evaluate whether the numerical datasets were normally distributed. Parametric tests (paired or unpaired t-test) were used when numerical datasets were normally distributed. On the other hand, non-parametric tests (the Wilcoxon signed rank test or Mann-Whitney test) were used when numerical datasets were not normally distributed. Continuous data are expressed as medians and ranges or interquartile ranges (IQR, 25 to 75th percentile). We used ANOVA and a post hoc test (Tukey-Kramer) for multiple comparisons in histology and stage. The unpaired t-test was used for age distribution, Fisher's exact test for sex, histology and stage distribution, and the Chi-square test for myasthenia gravis distribution. The area under the receiver operating characteristic (ROC) curve [AUC; ranging between 0.5 (chance) and 1.0 (perfect discrimination or accuracy)] was measured to characterize the accuracy of the DNA methylation signature to discriminate TC from thymoma. Survival curves were estimated using the Kaplan-Meier method and were compared with the log-rank test.

All statistical analyses were performed using two software programs (SPSS, version 24.0; SPSS, Inc.) and JMP, version 12.2; SAS Institute Inc.). A P-value $<0.05$ was considered to indicate a statistically significant difference.

\section{Results}

Screening of aberrantly methylated CGI in tumor samples. We initially screened 7 TC and 8 B3 thymoma samples obtained from freshly frozen specimens (Table SI) with Illumina HumanMethylation450 K BeadChip to identify differentially methylated CGI in a genome-wide manner. Fig. S2 depicts a volcano plot of the differential CGI methylation profiles of thymoma and TC samples. The $\mathrm{x}$-axis indicates the average $\beta$-value difference (methylation level). The y-axis indicates the 
Table I. Characteristics of patients.

\begin{tabular}{|c|c|c|c|c|c|c|}
\hline \multirow[b]{2}{*}{ Variable and category } & \multirow{2}{*}{$\begin{array}{l}\text { No. of cases } \\
\text { Period }\end{array}$} & \multicolumn{2}{|c|}{ Percentage } & & & \\
\hline & & Mean & Media & & & \\
\hline Age & $28-84$ & 60.1 & 63 & & & \\
\hline \multicolumn{7}{|l|}{ Sex } \\
\hline Male & 20 & $(43.5)$ & & & & \\
\hline Female & 26 & $(56.5)$ & & & & \\
\hline \multicolumn{7}{|l|}{ WHO histological classification } \\
\hline Thymoma & 30 & $(65.2)$ & & & & \\
\hline A & 5 & $(10.9)$ & & & & \\
\hline $\mathrm{AB}$ & 2 & $(4.3)$ & & & & \\
\hline B 1 & 4 & $(8.7)$ & & & & \\
\hline B2 & 10 & $(21.7)$ & & & & \\
\hline B3 & 9 & $(19.6)$ & & & & \\
\hline Thymic carcinoma & $12^{\mathrm{a}}$ & $(26.1)$ & & & & \\
\hline NECTT & 4 & $(8.7)$ & & & & \\
\hline Typical carcinoid & 2 & $(4.3)$ & & & & \\
\hline Atypical carcinoid & 1 & $(2.2)$ & & & & \\
\hline \multirow[t]{3}{*}{ Small cell carcinoma } & 1 & $(2.2)$ & & & & \\
\hline & & & \multicolumn{4}{|c|}{ Masaoka-Koga staging } \\
\hline & & & \multicolumn{4}{|c|}{$\begin{array}{l}\text { Carcinoma } \\
\text { including }\end{array}$} \\
\hline Masaoka-Koga staging (TETs) & & & \multicolumn{2}{|c|}{ Thymoma } & & STT \\
\hline I & 11 & $(23.9)$ & 10 & $(33.3)$ & 1 & $(6.3)$ \\
\hline II & 17 & $(37.0)$ & 10 & $(33.3)$ & 7 & $(43.8)$ \\
\hline III & 8 & (17.4) & 4 & (13.3) & 4 & $(25.0)$ \\
\hline IVA & 5 & $(10.9)$ & 4 & (13.3) & 1 & $(6.3)$ \\
\hline IVB & 5 & $(10.9)$ & 2 & $(6.7)$ & 3 & $(18.8)$ \\
\hline Myasthenia gravis & 9 & (19.6) & & & & \\
\hline
\end{tabular}

${ }^{\mathrm{a} O n e}$ case of combined thymic carcinoma and type B2 thymoma. TET, thymic epithelial tumor; NECTT, neuroendocrine tumor of the thymus.

- $\log 10$ value of the adjusted Welch's test P-value for each CGI. Red points are significant methylated $\mathrm{CpG}$ sites determined by the Bonferroni's test. The plots on the right show more methylated CGI in TC than in B3 thymoma (Fig. S2, arrow), while those on the left show more methylated CGI in B3 thymoma than in TC.

In total, 92 CGI were identified as differentially hypermethylated in the TC samples in relation to the B3 thymoma samples [FDR $<0.05$ and $\beta$-difference (TC-B3 thymoma) $>0.5$ ]. Table SIII shows the top 29 CGI significantly hypermethylated in TCs in relation to B3 thymomas. We investigated whether the DNA methylation of the 29 genes was related to cancer using the PubMed database and selected $G$ protein subunit gamma 4 (GNG4), growth hormone secretagogue receptor (GHSR), homeobox D9 (HOXD9) and spalt like transcription factor 3 (SALL3).

CGI methylation status of GHSR, GNG4, HOX9 and SALL3 between TCs and B3 thymomas in an Illumina HumanMethylation450 K BeadChip array. A schematic diagram of the GHSR structure and $\mathrm{CpG}$ sites around exon 1 is presented in Fig. 1A. Using an Illumina HumanMethylation450 K BeadChip array, CGI including $107 \mathrm{CpG}$ sites within the GHSR gene was the 16th CGI significantly hypermethylated in TCs in relation to B3 thymomas (Table SIII). CpG sites within CGI in TCs exhibited higher levels of methylation than in B3 thymomas. Two CGI regions (from cg10109500 to cg17152757 and from cg04851268 to cg06737494) exhibited significantly higher methylation levels in the TC samples ( $\mathrm{P}<0.01$; Fig. 1A). A schematic diagram of the GNG4 structure is shown in Fig. 1B. CGI, including $203 \mathrm{CpG}$ sites within the GNG4 gene was the 7th CGI significantly hypermethylated in TCs in relation to type B3 thymomas (Table SIII). The DNA methylation rate of CpG sites from cg17816394 to cg06173536 within CGI in TCs was significantly higher than that in $\mathrm{B} 3$ thymomas $(\mathrm{P}<0.01$; Fig. 1B). A schematic diagram of the HOXD9 structure is shown in Fig. 1C. CGI, including $172 \mathrm{CpG}$ sites within the HOXD9 gene was the 23th CGI significantly hypermethylated in TCs in relation to type B3 thymomas (Table SIII). The DNA methylation rate of $\mathrm{CpG}$ sites from cg23068499 to cg11597131 within 
A
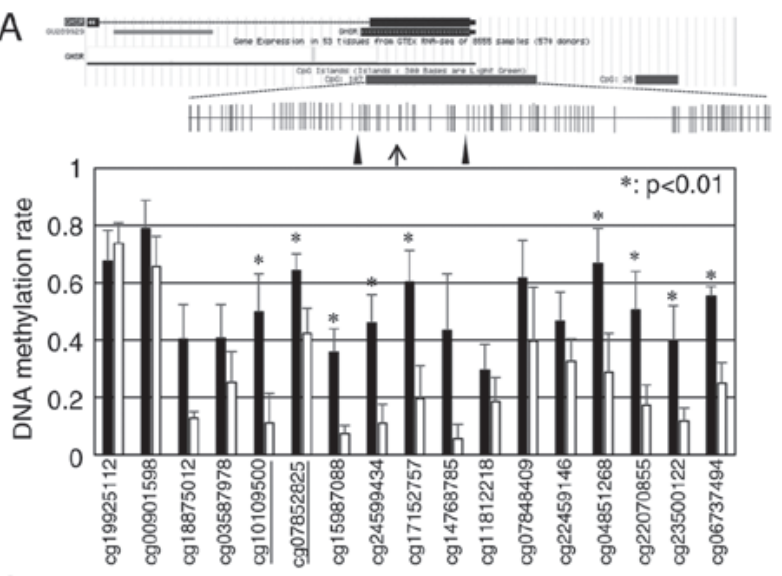

C
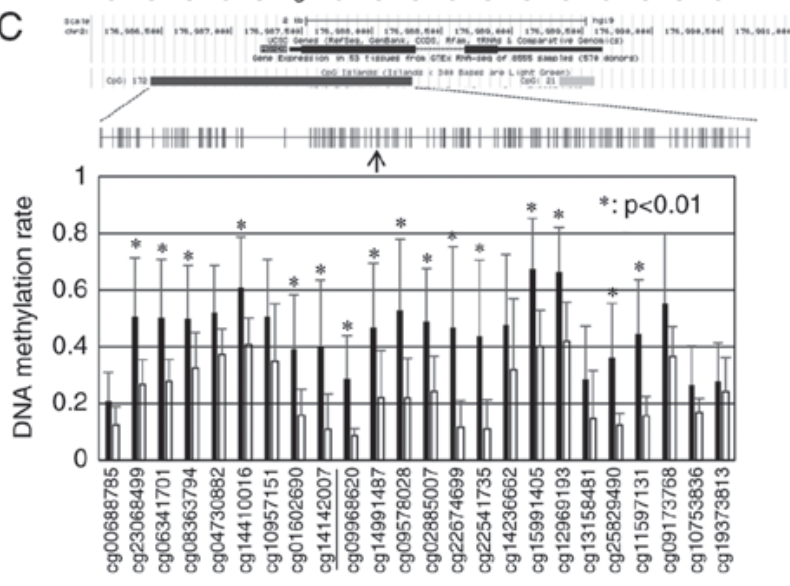

B
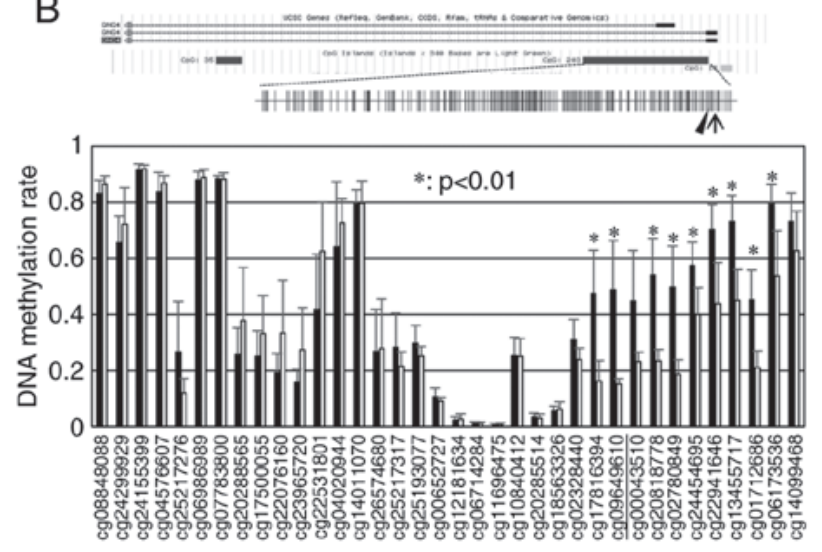

$\mathrm{D}$
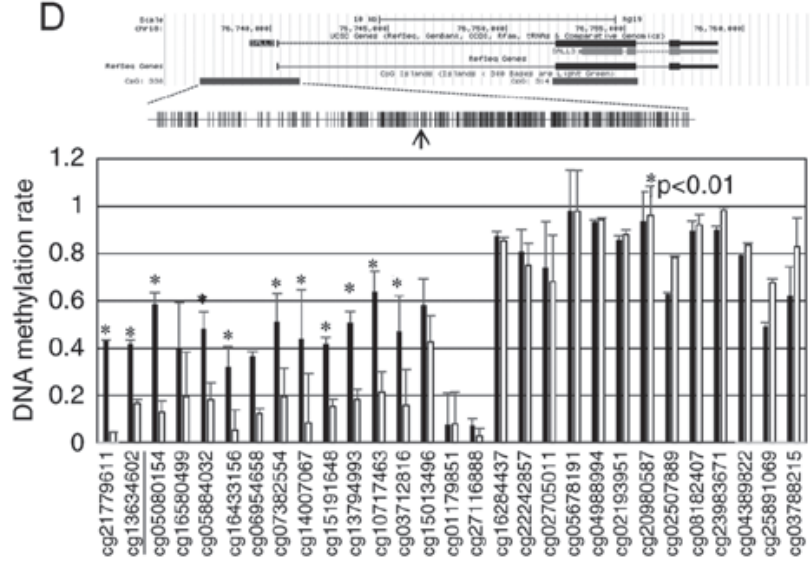

Figure 1. Schematic diagram of 4 genes. (A) Schematic diagram of the GHSR structure. Vertical bars indicate the positions of CpG dinucleotides. The vertical scale indicates average $\beta$-values of the methylation level of each $\mathrm{CpG}$ site between TC (black bar) and B3 thymoma (white bar). The arrowheads show the CpG sites of $\operatorname{cg} 10109500$ and 07852825 and the arrow shows $\mathrm{CpG}$ sites $(+242,+249,+251,+257$, and +259 from the transcription start site) examined by quantitative pyrosequencing. The asterisk shows $\mathrm{CpG}$ sites with a significantly different $(\mathrm{P}<0.01)$ methylation rate between $\mathrm{TC}$ and thymoma. (B) schematic diagram of the GNG4 structure. The vertical scale indicates average $\beta$-values of the methylation level of each CpG site between TC (black bar) and B3 thymoma (white bar). Arrowheads show the $\mathrm{CpG}$ site of cg09649610 and the arrow shows $\mathrm{CpG}$ sites examined by quantitative pyrosequencing. The asterisk shows a CpG site with a significantly different $(\mathrm{P}<0.01)$ methylation rate between $\mathrm{TC}$ and thymoma. (C) Schematic diagram of the HOXD9 structure. A vertical scale indicating average $\beta$-values of the methylation level of each $\mathrm{CpG}$ site between TC (black bar) and B3 thymoma (white bar) are indicated. The arrow shows the CpG site of cg14142007 and CpG sites examined by quantitative pyrosequencing. The asterisk shows a CpG site with a significantly different (P<0.01) methylation rate between TC and thymoma. (D) Schematic diagram of the SALL3 structure. The vertical scale indicates average $\beta$-values of the methylation level of each CpG site between TC (black bar) and B3 thymoma (white bar). The arrow shows the CpG site of cg13634602 and CpG sites examined by quantitative pyrosequencing. The asterisk shows a $\mathrm{CpG}$ site with a significantly different $(\mathrm{P}<0.01)$ methylation rate between $\mathrm{TC}$ and thymoma.

CGI in TCs was significantly higher than that in B3 thymomas $(\mathrm{P}<0.01)$. A schematic diagram of the SALL3 structure is presented in Fig 1D. CGI, including $338 \mathrm{CpG}$ sites within the SALL3 gene was the 26th CGI significantly hypermethylated in TCs in relation to type B3 thymomas (Table SIII). The DNA methylation rate of $\mathrm{CpG}$ sites from $\operatorname{cg} 21779611$ to $\operatorname{cg} 03712816$ within CGI in TCs was significantly higher than that in B3 thymomas $(\mathrm{P}<0.01)$.

CGI methylation status of GHSR, GNG4, HOX9 and SALL3 in TETs and paired thymic samples in pyrosequencing. To confirm the data obtained using Illumina HumanMethylation $450 \mathrm{~K}$ BeadChip, we examined the DNA methylation of CpG sites $(+242,+249,+251,+257$ and +259 from the transcription start site) of GHSR between $\operatorname{cg} 10109500(+350)$ and $\operatorname{cg} 07852825$ (+52) using pyrosequencing (Fig. 1A). Moskalev et al reported that $\mathrm{CpG}$ sites in this region frequently exhibited a higher DNA methylation in various cancers than in healthy tissue (11). Fig. 2A shows the association for the DNA methylation rate of $5 \mathrm{CpG}$ sites in the GHSR gene between thymoma and the thymus. The DNA methylation rate was significantly higher for thymoma than for the thymus (paired t-test, $\mathrm{P}=0.003$ ). Fig. 2A also shows the association for the DNA methylation rate of the GHSR gene between TC and the thymus. The DNA methylation rate was significantly higher for TC than for the thymus (paired t-test, $\mathrm{P}=0.0003$ ).

We examined the DNA methylation of $\mathrm{CpG}$ sites of GNG4 around $\operatorname{cg} 09649610$ (+350 from TSS) using pyrosequencing (Fig. 1B). Pal et al previously reported that CpG sites in this region frequently exhibited higher DNA methylation in glioblastoma than in healthy tissue (12). As shown in Fig. 2B, no significant differences in the DNA methylation rate of the GNG4 gene were observed between thymoma and the thymus (paired t-test, $\mathrm{P}=0.176$ ). Fig. $2 \mathrm{~B}$ also shows that the DNA methylation rate was significantly higher for TC than for the thymus (Wilcoxon signed rank test, $\mathrm{P}=0.018$ ).

We examined the DNA methylation of $\mathrm{CpG}$ sites of HOXD9 around cg14142007 (-753 from TSS) using pyrosequencing (Fig. 1C). Marzese et al reported that $\mathrm{CpG}$ sites in 

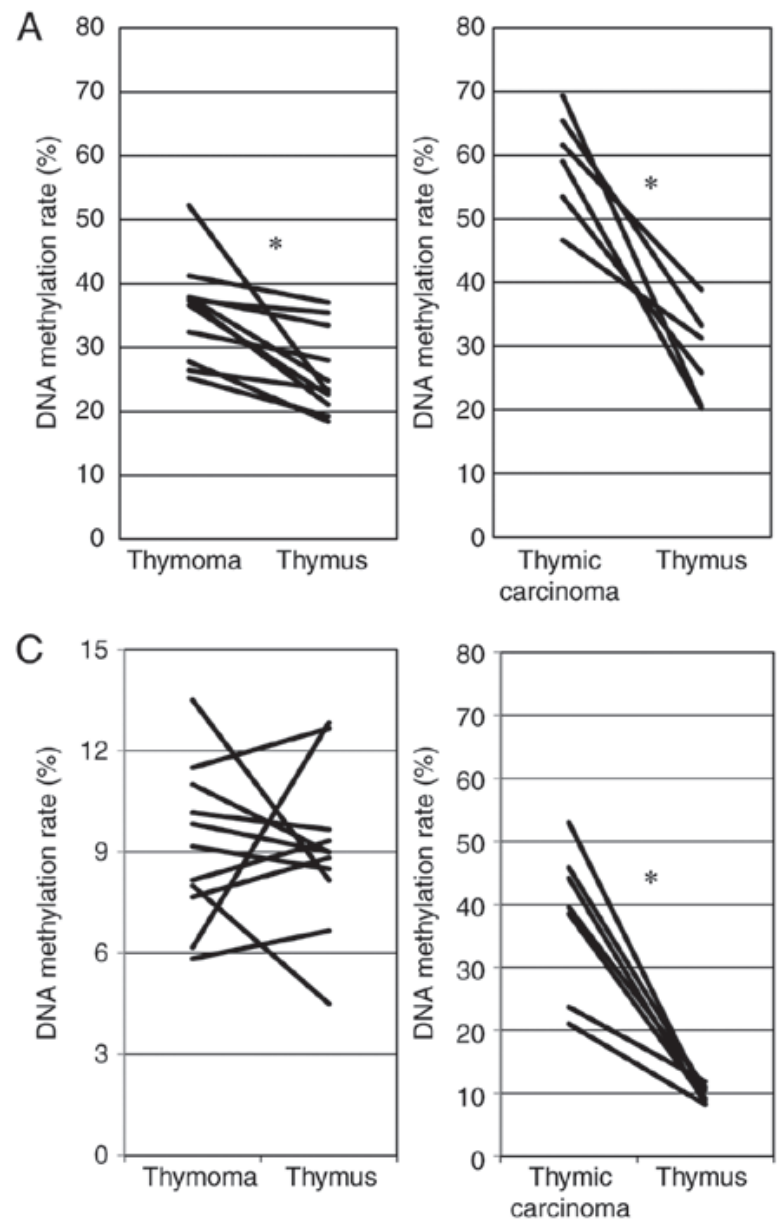
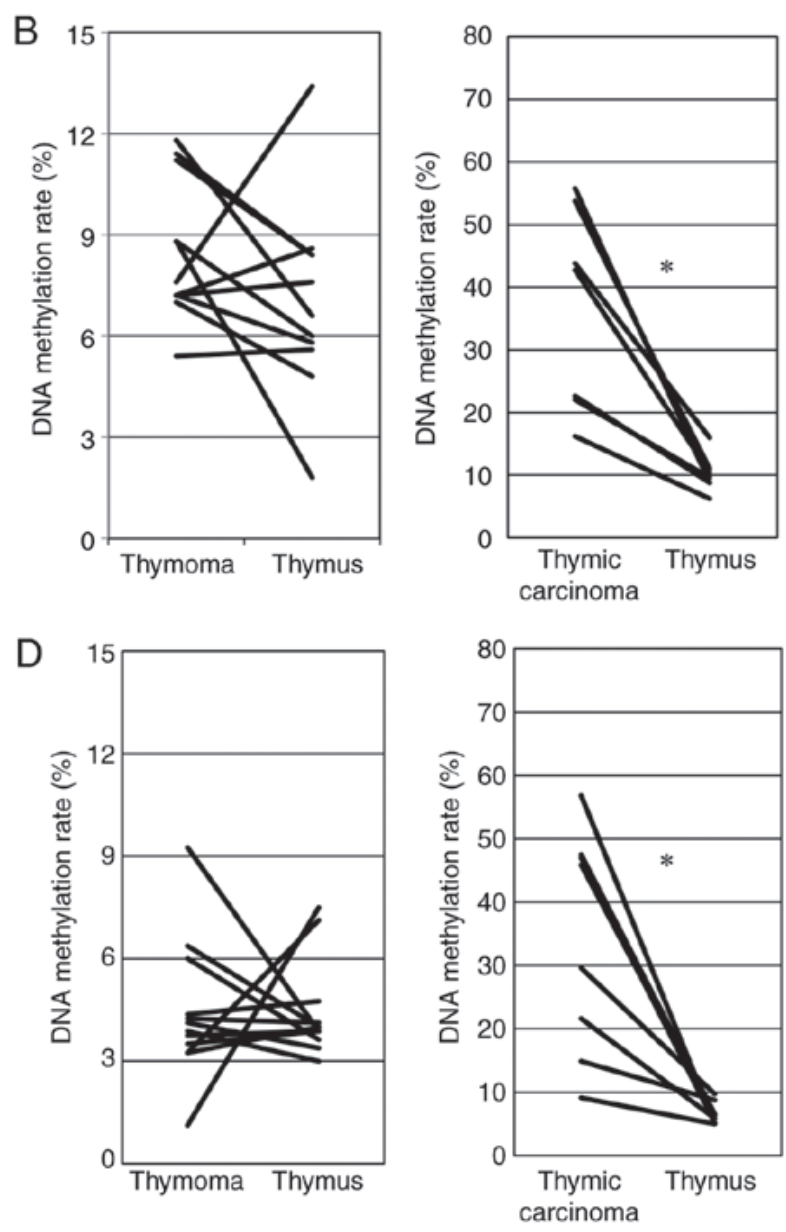

Figure 2. DNA methylation rate of 4 genes in tumors and paired thymic tissues. (A) DNA methylation rate of the GHSR gene in thymomas and paired thymic tissues and in thymic carcinoma and paired thymic tissues. Average DNA methylation values (percentages) of quantitative pyrosequencing in 11 thymomas and paired thymic tissues. Average DNA methylation values of quantitative pyrosequencing in 6 thymic carcinoma and paired thymic tissues. Samples from the same patient are linked with straight lines. (B) DNA methylation rate of the GNG4 gene in thymomas and paired thymic tissues and in thymic carcinoma and paired thymic tissues. Average DNA methylation values of quantitative pyrosequencing in 11 thymomas and paired thymic tissues. Average DNA methylation values of quantitative pyrosequencing in 7 thymic carcinoma and paired thymic tissues. (C) DNA methylation rate of the HOXD9 gene in thymomas and paired thymic tissues and in thymic carcinoma and paired thymic tissues. Average DNA methylation values of quantitative pyrosequencing in 11 thymomas and paired thymic tissues. Average DNA methylation values of quantitative pyrosequencing in 7 thymic carcinoma and paired thymic tissues. (D) DNA methylation rate of the SALL3 gene in thymomas and paired thymic tissues in thymic carcinoma and paired thymic tissues. Average DNA methylation values of quantitative pyrosequencing in 12 thymomas and paired thymic tissues. Average DNA methylation values of quantitative pyrosequencing in 8 thymic carcinoma and paired thymic tissues. " $\mathrm{P}<0.05$, as indicated.

this region frequently exhibited higher DNA methylation in malignant melanoma with metastasis than in healthy tissue and malignant melanoma without metastasis (13). As shown in Fig. 2C, no significant differences in the DNA methylation rates of the HOXD9 gene were observed between thymoma and the thymus (paired t-test, $\mathrm{P}=0.861$ ). Fig. $2 \mathrm{C}$ also shows that the DNA methylation rate was significantly higher for TC than for the thymus (Wilcoxon signed rank test, $\mathrm{P}=0.018$ ).

We examined the DNA methylation of $\mathrm{CpG}$ sites of the SALL3 gene around cg13634602 (-1095 from TSS) using pyrosequencing. Misawa et al reported that $\mathrm{CpG}$ sites from -319 to 184 frequently showed DNA methylation in head and neck cancer (14). In this study, we attempted to create pyrosequence primers, but were unsuccessful; therefore, we created suitable primers for PCR and sequencing on the upstream side $(-1,095)$. As shown in Fig. 2D, no significant differences in the DNA methylation rate of the SALL3 gene were observed between thymoma and the thymus (Wilcoxon signed rank test,
$\mathrm{P}=0.906)$. Fig. $2 \mathrm{D}$ also shows that the DNA methylation rate was significantly higher for TC than for the thymus (Wilcoxon signed rank test, $\mathrm{P}=0.0117$ ).

CGI methylation status of GHSR, GNG4, HOX9 and SALL3 in the pyrosequencing of TETs according to the WHO histological classification. Fig. 3A shows the median DNA methylation rate of the GHSR gene in TETs according to the WHO histological classification. The median DNA methylation rates in $\mathrm{A}+\mathrm{AB}+\mathrm{B} 1, \mathrm{~B} 2, \mathrm{~B} 3$ and TCs + NECTT were 32.4, 36.5, 38.0 and 55.4, respectively. The median DNA methylation rate was significantly higher for TCs including NECTT than for thymoma (55.4 vs. 36.5) (unpaired t-test, $\mathrm{P}<0.001$ ). No significant differences were observed in the median DNA methylation rate according to the WHO histological classification for thymomas $(\mathrm{A}+\mathrm{AB}+\mathrm{B} 1$ vs. B2, B2 vs. B3, and $\mathrm{A}+\mathrm{AB}+\mathrm{B} 1$ vs. B3, ANOVA and Tukey-Kramer tests). The median DNA methylation rate was significantly higher for TCs than for each group of thymoma $(\mathrm{A}+\mathrm{AB}+\mathrm{B} 1, \mathrm{~B} 2$ and $\mathrm{B} 3$, 

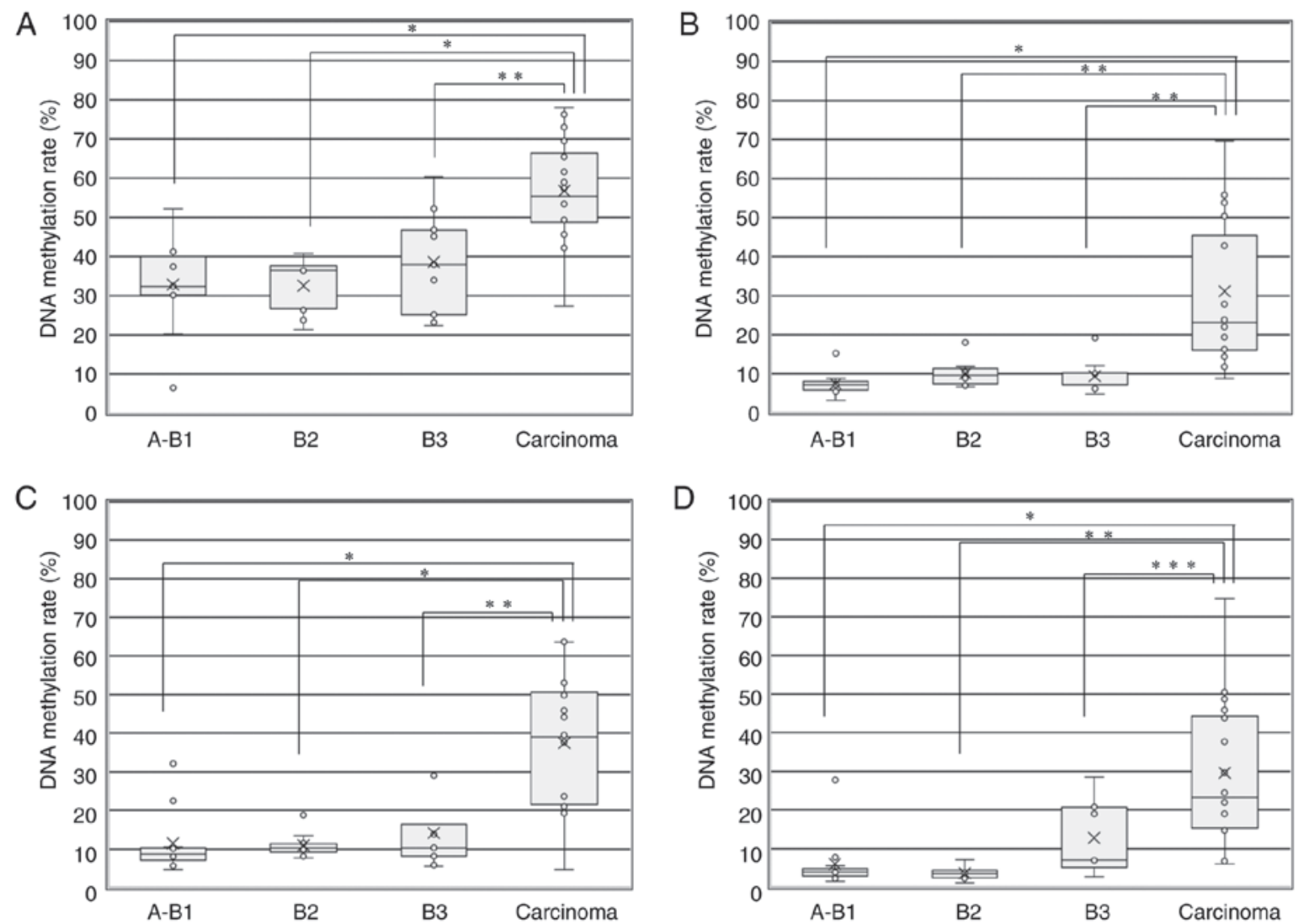

Figure 3. DNA methylation rate of 4 genes in TETs according to the WHO histological classification. (A) DNA methylation rate of the GHSR gene in TETs according to the WHO histological classification (A+AB+B1, B2, B3 and TCs + NECTT). The upper and lower ends of the whiskers, the upper and lower edges of the boxes, the horizontal lines across each box, ' $\mathrm{x}$ ' marks and the circles outside the boxes represent the upper and lower extremes, the upper (75th) and lower (25th) quartiles, medians, means and data outliers, respectively. Median DNA methylation rates in A+AB+B1, B2, B3, and TCs + NECTT were 32.4 (range, 6.6-52.2; IQR, 30.2-40), 36.5 (range, 21.4-40.8; IQR, 26.8-37.7), 38.0 (range, 22.4-60.4; IQR, 25.2-46.8) and 55.4 (range, 27.4-78.0; IQR, 48.7-66.4), respectively. The DNA methylation rate of the GHSR gene between TCs + NECTT and thymomas (A+AB+B1+B2+B3) was 55.4 (range, 27.4-78.0; IQR, 48.7-66.4) vs. 36.5 (range, 6.6-60.4; IQR, 26.8-40.3). The median DNA methylation rate was significantly higher for TCs than for each group of thymoma (ANOVA: $\mathrm{P}<0.0001$ and Tukey-Kramer tests: $\mathrm{A}+\mathrm{AB}+\mathrm{B} 1$ vs. carcinoma, $\mathrm{P}<0.0001$; B2 vs. carcinoma, $\mathrm{P}<0.0001$; $\mathrm{B} 3$ vs. carcinoma, $\mathrm{P}=0.0049$ ). (B) $\mathrm{DNA}$ methylation rate of the GNG4 gene in TETs according to the WHO histological classification (A+AB+B1, B2, B3 and TCs + NECTT). Median DNA methylation rates in $\mathrm{A}+\mathrm{AB}+\mathrm{B} 1, \mathrm{~B} 2$, B3, and TCs + NECTT were 7.2 (range, 3.2-15.2; IQR, 5.8-8.0), 9.6 (range, 6.6-18.0; IQR, 7.5-11.4), 7.2 (range, 4.8-19.2; IQR, 7.2-10.2) and 23.2 (range, 8.8-69.6; IQR, 16.1-45.5), respectively. The DNA methylation rate of the GNG4 gene between TCs + NECTT and thymomas was 23.2 (range, 8.8-69.6; IQR, 16.1-45.5) vs. 7.7 (range, 3.2-19.2; IQR, 6.7-10.4). The median DNA methylation rate was significantly higher for TCs than for each group of thymoma (ANOVA: $\mathrm{P}<0.0001$ and Tukey-Kramer tests: $\mathrm{A}+\mathrm{AB}+\mathrm{B} 1$ vs. carcinoma, $\mathrm{P}<0.0001$; $\mathrm{B} 2$ vs. carcinoma, $\mathrm{P}=0.0003$; $\mathrm{B} 3$ vs. carcinoma, $\mathrm{P}=0.0003)$. (C) DNA methylation rate of the HOXD9 gene in TETs according to the WHO histological classification $(\mathrm{A}+\mathrm{AB}+\mathrm{B} 1, \mathrm{~B} 2, \mathrm{~B} 3$ and TCs $+\mathrm{NECTT})$ The median DNA methylation rates in A+AB+B1, B2, B3, and TCs + NECTT were 8.7 (range, 4.7-32.2; IQR, 7.1-10.3), 10.3 (range, 7.7-18.8; IQR, 9.2-11.4), 10.3 (range, 5.5-30.0; IQR, 8.2-16.3) and 39.0 (range, 4.7-63.7; IQR, 21.5-50.6), respectively. The DNA methylation rate of the HOXD9 gene between TCs + NECTT and thymomas was 39.0 (range, 4.7-63.7; IQR, 21.5-50.6) vs. 9.6 (range, 4.7-32.2; IQR, 8.1-13.0). The median DNA methylation rate was significantly higher for TCs than for each group of thymoma (ANOVA: $\mathrm{P}<0.0001$ and Tukey-Kramer tests: $\mathrm{A}+\mathrm{AB}+\mathrm{B} 1$ vs. carcinoma, $\mathrm{P}<0.0001 ; \mathrm{B} 2$ vs. carcinoma, $\mathrm{P}<0.0001 ; \mathrm{B} 3$ vs. carcinoma, $\mathrm{P}=0.0002)$. (D) DNA methylation rate of the $S A L L 3$ gene in TETs according to the WHO histological classification $(\mathrm{A}+\mathrm{AB}+\mathrm{B} 1, \mathrm{~B} 2, \mathrm{~B} 3$ and TCs + NECTT). The median DNA methylation rates in A+AB+B1, B2, B3, and TCs + NECTT were 3.9 (range, 1.5-27.8; IQR, 2.9-4.8), 3.5 (range, 1.1-7.1; IQR, 2.4-4.4), 7.0 (range, 2.8-28.5; IQR, 5.1-20.8) and 23.3 (range, 6.0-74.8; IQR 15.3-44.3), respectively. The DNA methylation rate of the SALL3 gene between TCs + NECTT and thymomas was 23.3 (range, 6.0-74.8; IQR, 15.3-44.3) vs. 4.2 (range, 1.1-28.5; IQR, 3.1-7.0). The median DNA methylation rate was significantly higher for TCs than for each group of thymoma (ANOVA: $\mathrm{P}<0.0001$ and Tukey-Kramer tests: $\mathrm{A}+\mathrm{AB}+\mathrm{B} 1$ vs. carcinoma, $\mathrm{P}<0.0001 ; \mathrm{B} 2$ vs. carcinoma, $\mathrm{P}=0.0001$; $\mathrm{B} 3$ vs. carcinoma, $\mathrm{P}=0.0138$ ). TET, thymic epithelial tumor; $\mathrm{TC}$, thymic carcinoma; $\mathrm{NECTT}$, neuroendocrine tumor of the thymus. ${ }^{*} \mathrm{P}<0.0001,{ }^{* *} \mathrm{P}<0.001,{ }^{* * *} \mathrm{P}<0.005$, as indicated.

ANOVA and Tukey-Kramer tests) $(\mathrm{P}<0.001)$. We examined the accuracy of the methylation signature of 4 genes for the detection of TCs using a ROC curve analysis. We used AUC as the criterion of accuracy, which may range in value from 0.5 (chance) to 1.0 (perfect discrimination or accuracy). Fig. 4A shows the ROC curve for the accuracy of the GHSR methylation signature for TC detection from all tumors. It revealed high degrees of sensitivity and specificity for discriminating TCs and thymomas (AUC $=0.908$ ).
Fig. 3B shows the median DNA methylation rate of $5 \mathrm{CpG}$ sites in the GNG4 gene in TETs according to the WHO histological classification. The median DNA methylation rates in $\mathrm{A}+\mathrm{AB}+\mathrm{B} 1, \mathrm{~B} 2, \mathrm{~B} 3$ and TCs + NECTT were 7.2, 9.6, 7.2 and 23.2, respectively. The median DNA methylation rate was significantly higher for TCs, including NECTT than for thymoma (23.2 vs. 7.7). No significant differences were observed in the median DNA methylation rate according to the WHO histological classification of thymomas (ANOVA 

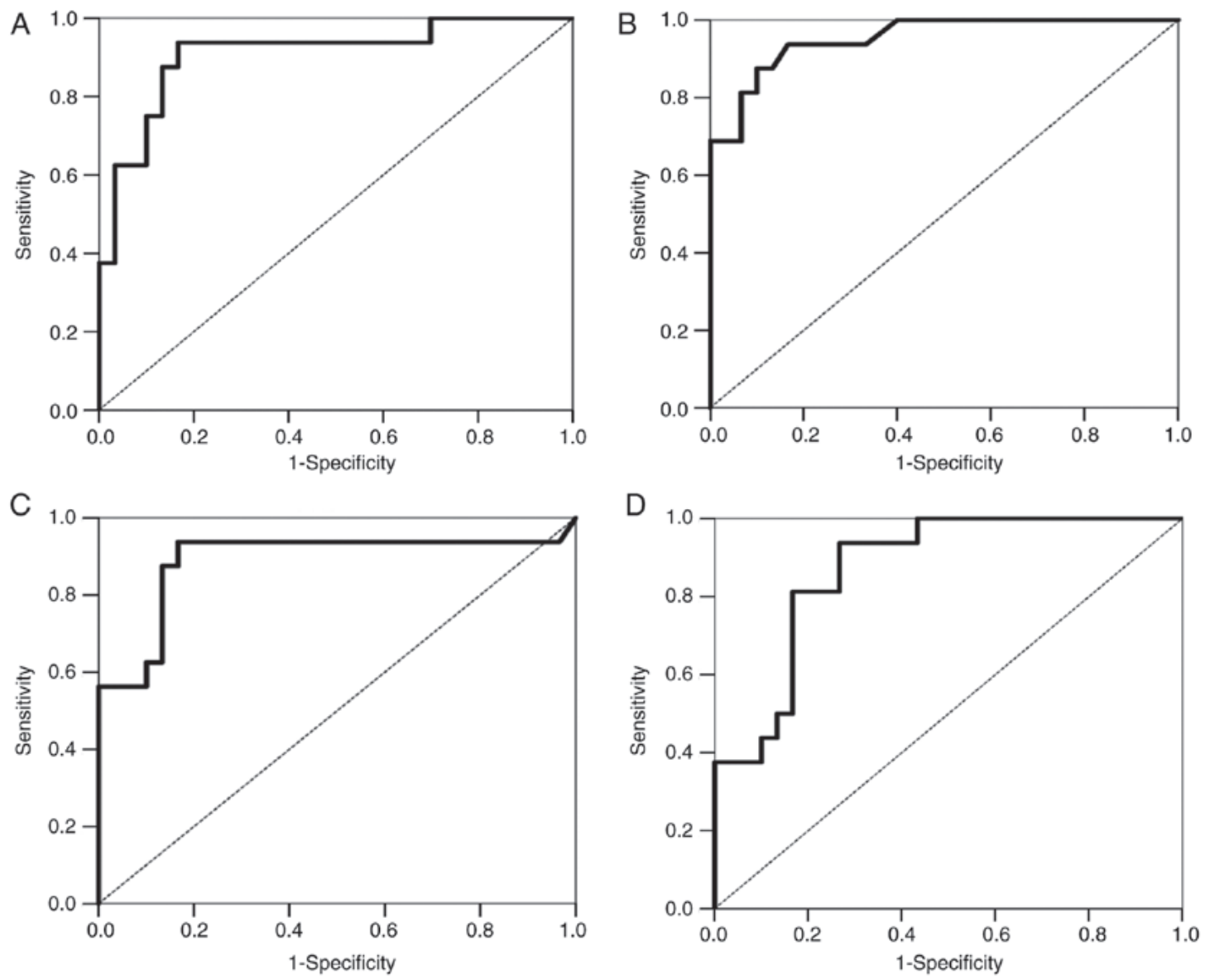

Figure 4. ROC curve for the accuracy of the methylation signature for TC detection from all tumors. (A) ROC curve using GHSR methylation. (B) ROC curve using GNG4 methylation. (C) ROC curve using HOXD9 methylation. (D) ROC curve using SALL3 methylation.

and Tukey-Kramer tests). The median DNA methylation rate was significantly higher for TCs than for each group of thymoma (ANOVA and Tukey-Kramer tests). Fig. 4B shows the ROC curve for the accuracy of the GNG4 methylation signature for TC detection from all tumors. It revealed high degrees of sensitivity and specificity for discriminating TCs and thymomas (AUC $=0.953$ ).

Fig. 3C shows the median DNA methylation rate of $5 \mathrm{CpG}$ sites in the HOXD9 gene in TETs according to the WHO histological classification. The median DNA methylation rates in $\mathrm{A}+\mathrm{AB}+\mathrm{B} 1, \mathrm{~B} 2, \mathrm{~B} 3$, and TCs + NECTT were 8.7, 10.3, 10.3 and 39.0, respectively. The median DNA methylation rate was significantly higher for TCs, including NECTT than for thymoma (39.0 vs. 9.6). No significant differences were observed in the mean DNA methylation rate according to the WHO histological classification of thymomas (ANOVA and Tukey-Kramer tests). The median DNA methylation rate was significantly higher for TCs than for each group of thymoma (ANOVA and Tukey-Kramer tests). Fig. 4C shows the ROC curve for the accuracy of the HOXD9 methylation signature for TC detection from all tumors. It revealed high degrees of sensitivity and specificity for discriminating TCs and thymomas $(\mathrm{AUC}=0.889)$.

Fig. 3D shows the median DNA methylation rate of $5 \mathrm{CpG}$ sites in the SALL3 gene in TETs according to the WHO histological classification. The median DNA methylation rates in $\mathrm{A}+\mathrm{AB}+\mathrm{B} 1, \mathrm{~B} 2, \mathrm{~B} 3$, and $\mathrm{TCs}+\mathrm{NECTT}$ were 3.9,
3.5, 7.0 and 23.3, respectively. The median DNA methylation rate was significantly higher for TCs including NECTT than for thymomas (23.3 vs. 4.2). No significant differences were observed in the median DNA methylation rate according to the WHO histological classification of thymomas (ANOVA and Tukey-Kramer tests). The median DNA methylation rate was significantly higher for TCs than for each group of thymomas (ANOVA and Tukey-Kramer tests). Fig. 4D shows the ROC curve for the accuracy of the SALL3 methylation signature for TC detection from all tumors. It revealed high degrees of sensitivity and specificity for discriminating TCs and thymomas (AUC=0.873).

CGI methylation status of GHSR, GNG4, HOX9 and SALL3 in pyrosequencing for TETs according to the Masaoka-Koga clinical stage. Fig. S3A shows the median DNA methylation rate of the GHSR gene in TETs according to the Masaoka-Koga clinical stage. The median DNA methylation rates in stages I, II, III, IVA and IVB were 32.4, 37.8, 39.8, 40.8 and 73.0, respectively. No significant differences were observed in the DNA methylation rate of the GHSR gene between each stage (ANOVA and Tukey-Kramer tests). Fig. S3B shows the median DNA methylation rate of the GNG4 gene in TETs according to the Masaoka-Koga clinical stage. The median DNA methylation rates in stages I, II, III, IVA and IVB were 7.6, 11.8, 10.2, 10.2 and 27.8, respectively. There was only significant difference between stage I and IVB (ANOVA and Tukey-Kramer 
tests). Fig. 3C shows the median DNA methylation rate of the HOXD9 gene in TETs according to the Masaoka-Koga clinical stage. The median DNA methylation rates in stages I, II, III, IVA and IVB were 10.2, 13.5, 14.8, 18.8 and 8.2, respectively. No significant differences were observed in the DNA methylation rate of the HOXD9 gene between each stage (ANOVA and Tukey-Kramer tests). Fig. 3D shows the median DNA methylation rate of the SALL3 gene in TETs according to the Masaoka-Koga clinical stage. The median DNA methylation rates in stages I, II, III, IVA and IVB were 5.0, 6.4, 9.3, 32.4 and 5.3, respectively. No significant differences were observed in the DNA methylation rate of the SALL3 gene between each stage (ANOVA and Tukey-Kramer tests).

Characteristics of patients grouped by the median value of each gene. Patients with TETs were divided into 2 groups according to the median value of the frequency of the DNA methylation of each gene. In total, 23 patients had a median value of the frequency of DNA methylation of the GHSR gene $>38.4$ (higher DNA methylation level), while that for the remaining 23 patients was $\leq 38.4$ (lower DNA methylation level). In total, 23 patients had a median value of the frequency of the DNA methylation of the GNG4 gene $>10.3$ (higher DNA methylation level), while that for the remaining 23 patients was $\leq 10.3$ (lower DNA methylation level). A total of 23 patients had a median value of the frequency of the DNA methylation of the HOX9 gene $>12.5$ (higher DNA methylation level), while that for the remaining 23 patients was $\leq 12.5$ (lower DNA methylation level). A total of 23 patients had a median value of the frequency of the DNA methylation of the SALL3 gene $>7.75$ (higher DNA methylation level), while that for the remaining 23 patients was $\leq 7.75$ (lower DNA methylation level). The characteristics of patients grouped by the median value of each gene are shown in Table II.

The proportion of patients with TC was significantly higher in the higher DNA methylation group $(65.2 \%)$ than in the low DNA methylation group $(4.3 \%, \mathrm{P}<0.001)$. No significant differences were observed in age, sex, the presence of $\mathrm{MG}$, or Masaoka-Koga staging between the 2 groups. There were 12 TETs without genes with DNA methylation, 12 TETs with 1 gene with DNA methylation, 2 TETs with 2 genes with DNA methylation, 4 TETs with 3 genes with DNA methylation, and 16 TETs with 4 genes with DNA methylation (data not shown).

Relapse-free survival curve of TETs with higher and lower levels of DNA methylation for GHSR, GNG4, HOX9 and SALL3. In all TET cases $(\mathrm{n}=46)$, the median follow-up time was 44.1 months (6.7-272.7 months). Two patients died from their tumors and one from another disease. A total of 16 patients had recurrence: Pleural dissemination in 9, lung metastasis in 4 , lymph node metastasis in 1 and multiple organ metastasis in 2 . In thymoma cases $(n=30)$, the median follow-up time was 48.4 months (6.7-272.7 months). One patient died from another disease. A total of 6 patients had recurrence: Pleural dissemination in 4 and lung metastasis in 2.

Fig. 5A shows the relapse-free survival curve of TETs with a higher level (>38.4) and lower level (s38.4) of DNA methylation in GHSR. A significant difference was observed in survival between the higher and lower level groups $(\mathrm{P}=0.029$, log-rank test). Fig. 5B shows the relapse-free survival curve of
TETs with a higher level (>10.3) and lower level ( $\leq 10.3)$ of DNA methylation in GNG4. A significant difference was observed in survival between the higher and lower level groups $(\mathrm{P}=0.002)$. Fig. 5C shows the relapse-free survival curve of TETs with a higher level $(>12.5)$ and lower level $(\leq 12.5)$ of DNA methylation in $H O X 9$. A significant difference was observed in survival between the higher and lower level groups $(\mathrm{P}=0.003)$. Fig. 5D shows the relapse-free survival curve of TETs with a higher level (>7.75) and lower level $(\leq 7.75)$ of DNA methylation in SALL3. A significant difference was observed in survival between the higher and lower level groups $(\mathrm{P}=0.014)$.

We examined the association between the number of genes with DNA methylation and relapse-free survival. TETs were divided into 3 groups: Tumors without a gene with DNA methylation, a tumor with 1 or 2 genes with DNA methylation, and a tumor with 3 or 4 genes with DNA methylation. Fig. 5E shows the relapse-free survival curve of TETs with 3-4 genes with DNA methylation, 1-2 genes with DNA methylation, and no genes with DNA methylation. A significant difference was observed in survival between TETs with 3-4 genes with DNA methylation and 1-2 genes with DNA methylation $(\mathrm{P}=0.031$, log-rank test) and between TETs with 3-4 genes with DNA methylation and no genes with DNA methylation $(\mathrm{P}=0.003)$.

In the thymoma cases, when the median value of the frequency of the DNA methylation of each gene divided thymomas into higher and lower level groups, no significant differences were observed in relapse-free survival between these groups for each gene (data not shown). However, when the mean value of the frequency of the DNA methylation of each gene divided thymomas into higher and lower level groups, a significant difference was noted in relapse-free survival between these groups for HOX9 and SALL3. The mean value (11.9) of the frequency of the DNA methylation of HOX9 was divided into higher and lower level groups. Fig. 5F shows the relapse-free survival curve of thymomas with higher and lower levels of DNA methylation in $H O X 9$. A significant difference was observed in survival between the higher and lower level groups $(\mathrm{P}=0.036)$. The mean value (9.56) of the frequency of the DNA methylation of SALL3 was divided into higher and lower level groups. Fig. 5G shows the relapse-free survival curve of thymomas with higher and lower levels of DNA methylation in SALL3. A significant difference was observed in survival between the higher and lower level groups $(\mathrm{P}=0.003)$.

\section{Discussion}

Thymoma exhibits a weak malignant behavior, while TC is a more aggressive and refractory tumor (1-3). Although surgery is the optimal treatment option for operable $\mathrm{TC}$, the treatment for advanced TC is limited to chemotherapy, which is generally not curative (1). The development of an optimal therapy for advanced TC has been hampered by insufficient knowledge on genetic and epigenetic alterations in TC (4). Recent studies have comprehensively examined genetic alterations using next generation sequencing (4-6). However, limited information is currently available on epigenetic alterations $(7,8)$. In this study, we performed the genome-wide screening of aberrantly methylated CGI in TETs and identified 92 CGI that were significantly hypermethylated in TC. We examined the 


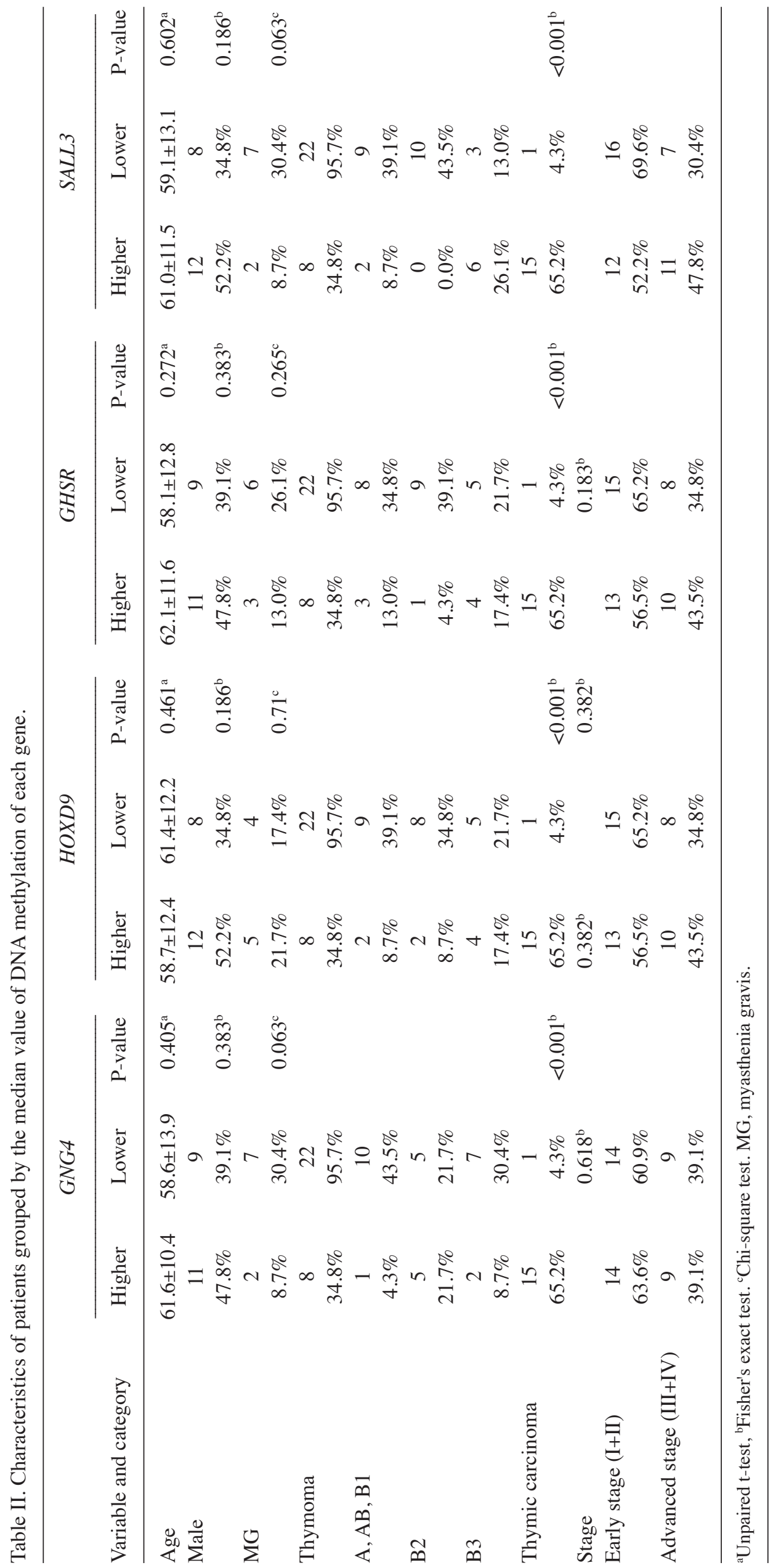



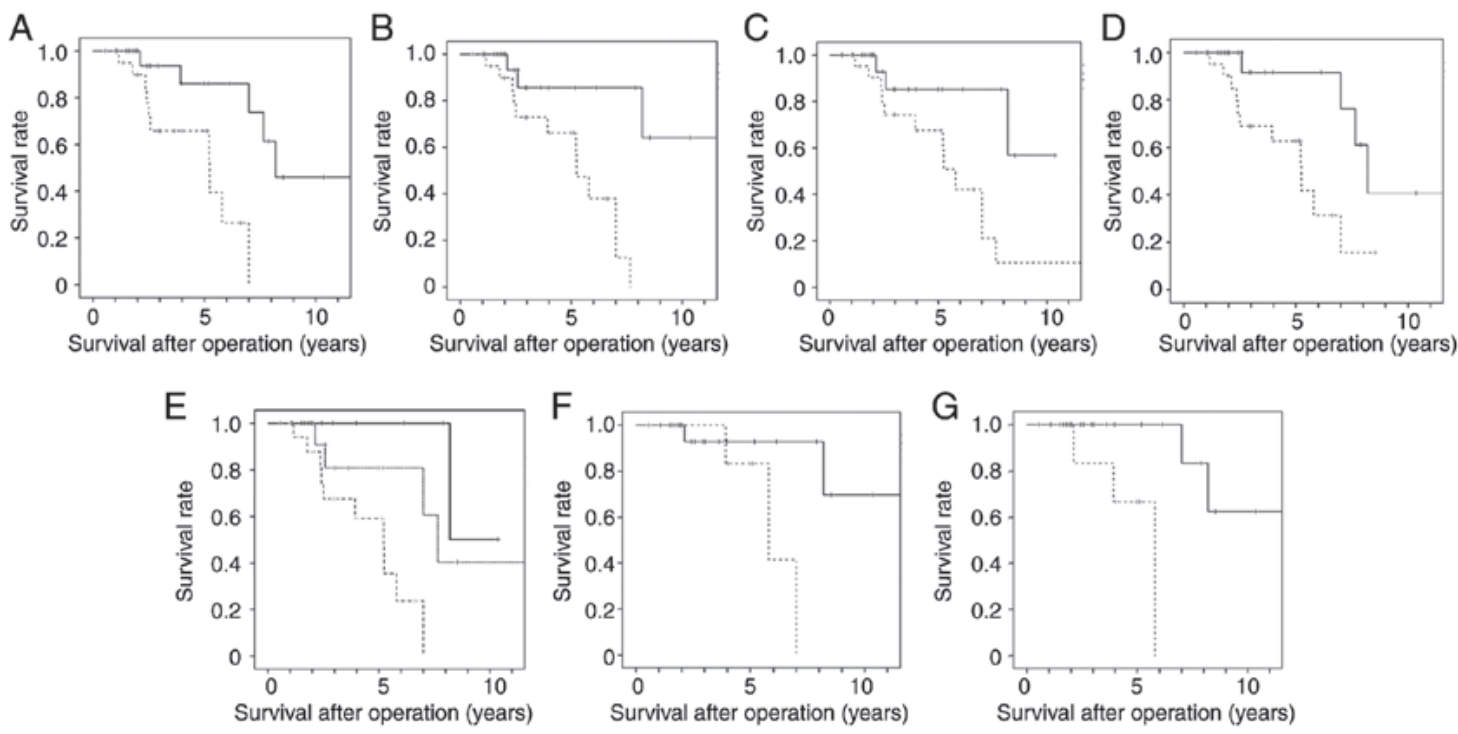

Figure 5. Survival curve of TETs with higher and lower levels of DNA methylation. (A) Relapse-free survival curve of TETs with higher and lower levels of DNA methylation in GHSR. The median value (38.4) of the frequency of the DNA methylation of GHSR was divided into higher (dotted line) and lower level groups (solid line). A significant difference was observed in survival between the higher and lower level groups ( $\mathrm{P}=0.029$, log-rank test). (B) Relapse-free survival curve of TETs with higher and lower levels of DNA methylation in GNG4. The median value (10.3) of the frequency of the DNA methylation of GNG4 was divided into higher (dotted line) and lower level groups (solid line). A significant difference was observed in survival between the higher and lower level groups ( $\mathrm{P}=0.002$, log-rank test). (C) Relapse-free survival curve of TETs with higher and lower levels of DNA methylation in HOXD9. The median value (12.5) of the frequency of the DNA methylation of HOXD9 was divided into higher (dotted line) and lower level groups (solid line). A significant difference was observed in survival between the higher and lower level groups ( $\mathrm{P}=0.003, \log$-rank test). (D) Relapse-free survival curve of TETs with higher and lower levels of DNA methylation in SALL3. The median value (7.75) of the frequency of the DNA methylation of SALL3 was divided into higher (dotted line) and lower level groups (solid line). A significant difference was observed in survival between the higher and lower level groups $(\mathrm{P}=0.014, \log$-rank test). (E) Relapse-free survival curve of TETs with 3 or 4 genes with DNA methylation, 1 or 2 genes with DNA methylation, and no genes with DNA methylation. A significant difference was observed in survival between TETs with 3 or 4 genes with DNA methylation (broken line) and 1 or 2 genes with DNA methylation (dotted line) $(\mathrm{P}=0.031$, log-rank test) and between TETs with 3 or 4 genes with DNA methylation (broken line) and no genes with DNA methylation (solid line) ( $\mathrm{P}=0.003$, log-rank test). (F) Relapse-free survival curve of thymomas with higher and lower levels of DNA methylation in HOX9. The mean value (11.9) of the frequency of the DNA methylation of $\mathrm{HOX}_{9}$ was divided into higher ( $\mathrm{n}=7$, dotted line) and lower level groups $(\mathrm{n}=21$, solid line). A significant difference was observed in survival between the higher and lower level groups ( $\mathrm{P}=0.036$, log-rank test). (G) Relapse-free survival curve of thymomas with higher and lower levels of DNA methylation in SALL3. The mean value (9.56) of the frequency of the DNA methylation of $S A L L 3$ was divided into higher (n=6, dotted line) and lower level groups ( $\mathrm{n}=22$, solid line). A significant difference was observed in survival between the higher and lower level groups $(\mathrm{P}=0.003$, log-rank test). TET, thymic epithelial tumor.

promoter methylation of GNG4, GHSR, HOXD9, and SALL3 in 46 TETs and 20 paired thymic samples using bisulfite pyrosequencing to identify a rational targeted therapy.

GHSR is a receptor of 'Ghrelin' that is involved in the modulation of functions, such as hormone secretion, energy balance and gastric acid release (15). GHSR encodes a member of the G-protein coupled receptor (GPCR) family and has 2 transcript variants, GHSR 1a and $1 \mathrm{~b}$ (16). Previous studies have demonstrated that GHSR is aberrantly hypermethylated in a number of cancers (e.g., lung, breast, prostate, pancreatic and colorectal cancers, glioblastoma, and B-cell chronic lymphocytic leukemia) and its methylation levels may be used to discriminate between cancer and healthy tissue, with GHSR hypermethylation being an early cancer event $(11,17,18)$. GPCR, comprising $\alpha, \beta$, and $\gamma$ subunits, responds to various extracellular stimuli, such as hormones, growth factors and sensory stimulating signals. GNG4 is one of fourteen $\gamma$ subunit proteins of GPCR (19). Pal et al reported that the promoter region of GNG4 was significantly hypermethylated and that its transcript level was significantly downregulated in glioblastoma and renal cell carcinoma (12). It functions as a tumor suppressor gene. Homeobox $(H O X)$ genes have $4 \mathrm{HOX}$ gene clusters: $H O X A, H O X B, H O X C$ and HOXD. HOXD 9 is a $H O X D$ gene that participates in the development and patterning of the forelimb and axial skeleton $(21,22)$. Previous studies have revealed that $H O X D 9$ promoter methylation is higher in tumors than in healthy tissue, and that DNA methylation levels correlate with the expression of HOXD 9 mRNA and protein in malignant melanoma and glioma $(13,23)$. Lv et al demonstrated that $H O X D 9$ was strongly expressed and functioned as an oncogene in hepatocellular carcinoma (24). SALL3 is one of 4 mammalian members of the sal-like (sall) gene family, which are involved in embryonic development (25). It encodes a C2H2-type zinc-finger protein (26). Recent studies have investigated the association between SALL3 expression and carcinogenesis in hepatocellular carcinoma, head and neck carcinoma and cervical carcinoma, and have demonstrated that it functions as a tumor suppressor gene; the hypermethylation of CGI in the promoter region of SALL3 reduced SALL3 mRNA levels (14,27-29).

The results of the present study revealed that promoter methylation was significantly higher in TC than in thymoma, and demonstrated highly discriminatory ROC profiles that clearly distinguished TCs from thymomas in all 4 genes. Furthermore, the promoter methylation of all 4 genes was higher in TC than that in the thymus. The DNA methylation of these 4 genes has been shown to be significantly higher in several types of cancer than in corresponding healthy tissues. GNG4 and 
SALL3 function as tumor suppressor genes (12,14,20,27-29) and HOXD9 acts as an oncogene (24). These genes are a common epigenetic alteration of high diagnostic value in TC. As shown in Table II, 15 (94\%) out of 16 thymic carcinomas had a high level of DNA methylation on each gene. The rate of diagnosis of thymic carcinoma was not more sensitive by combining the methylation of 4 genes. The characteristics and behavior of TC, but not thymoma, are similar to those of other types of cancer. This result indicated that the epigenetic pattern of TC significantly differed from that of thymoma. Recent comprehensive genetic analyses using next-generation sequencing have also revealed that the incidence of somatic non-synonymous mutations is significantly higher in TC than in thymomas (4-6). Clinically, TC entirely differs from thymoma from a pathological aspect, its malignant behavior, complications of autoimmune diseases and prognosis (1-3). Genetic and epigenetic differences between TC and thymoma may influence their clinical differences.

We propose two mechanisms based on the result that TC frequently exhibits a higher methylation of the promoter region of cancer-related genes than thymoma. In one mechanism, we found that SALL3 methylation was significantly higher in TC than in thymoma and the thymus. Shikauchi et al revealed that SALL3 binds to DNMT3A by a direct interaction between the double zinc finger motif of SALL3 and the PWWP domain of DNMT3A in hepatocellular carcinoma, and that SALL3 has the ability to inhibit DNMT3A-mediated DNA methylation (28). We thus hypothesized that SALL3 promoter methylation reduces SALL3 protein levels, which, in turn, inhibits methylation promotion by DNMT3A. Reduced SALL3 protein levels enhance DNMT3A activity and the CGI of cancer-related genes are hypermethylated in TC. In the other mechanism, Wang et al revealed that the incidence of somatic non-synonymous mutations of epigenetic regulatory genes (chromatin remodeling, histone modifications and DNA methylation) was significantly higher in TC (38\%) than in thymoma (10\%) (4). Mutated epigenetic regulatory genes in TC may induce higher levels of the promoter methylation of cancer-related genes. A clearer understanding of the mechanisms through which alterations in epigenetic regulation play a role in $\mathrm{TC}$ will contribute to the future tailoring of drugs to tumors with specific epigenetic alterations.

Although GHSR promoter methylation was significantly higher in thymoma than in the thymus, no significant differences were observed in promoter methylation for the other 3 genes. Furthermore, no significant differences were noted in the methylation of genes among the thymoma subtypes. These results suggest that epigenetic alterations in the 3 genes are not involved in the tumorigenesis of thymoma. The frequency of GHSR methylation increased in the order of the thymus, thymoma and TC. Jandaghi et al reported that GHSR hypermethylation is a pan-cancer marker regardless of the tissue from which the tumor originates (18). GHSR may be involved in TC and thymoma. The promoter methylation of the 4 genes was not significantly higher in advanced-stage tumors (III and IV) than in early-stage tumors (I and II) in all TETs. Moskalev et al revealed no significant differences in GHSR hypermethylation between the early and advanced stages of lung, breast, and pancreatic cancers (11). These findings suggest that the hypermethylation of cancer-related genes is an early cancer event.

Promoter methylation is related to malignant behavior and the relapse-free survival of tumors. Since there were a few deaths due to tumors, we used the relapse-free survival of tumors as a prognostic factor. In all 4 genes, relapse-free survival was significantly worse in tumors with a higher DNA methylation than in those with a lower DNA methylation in all TETs. Moreover, relapse-free survival was significantly worse in thymomas with a higher DNA methylation of HOXD9 or SALL3 than in those with a lower DNA methylation. Marzese et al revealed that patients with HOXD 9 hypermethylation in malignant melanoma had a poorer disease-free and overall survival (13). SALL3 methylation was identified as an independent predictor of poor survival in head and neck cancer (14). Relapse-free survival was worse in tumors with more genes with higher DNA methylation than in those with less genes with higher DNA methylation (Fig. 5E). These findings suggest that the relapse-free survival in TETs is related to the combination of the methylation of 4 genes.

There were some limitations to the present study. We examined the promoter methylation of GHSR, GNG4, HOX9 and SALL3 in 46 TETs and 20 thymic samples using bisulfite pyrosequencing. TC and NECT case numbers were lower (12 for TC and 4 for NECTT) as these tumors are very rare. Thymomas are stratified into 5 entities (types A, AB, B1, B2 and B3) based on the morphology of epithelial cells and the lymphocyte-to-epithelial cell ratio, and the ratio of lymphocytes to tumor cells is high in AB, B1 and B2 thymomas. Since we were unable to separate tumor cells from lymphocytes prior to DNA extraction, the presence of lymphocytes in resected $\mathrm{AB}, \mathrm{B} 1$ and $\mathrm{B} 2$ thymomas may have influenced the promoter methylation rate.

In conclusion, promoter methylation was significantly higher in TC than in thymoma and the thymus and exhibited high discrimination between TC and thymoma in all 4 genes. As regardsall 4 genes, relapse-free survival was significantly worse in tumors with a higher DNA methylation than in those with a lower DNA methylation in all TETs. The combination of 4 genes was not more sensitive than the individual genes alone for diagnosis, but may be superior for prognosis. These genes are a common epigenetic alterations of high diagnostic value in TC, which may be involved in the carcinogenesis of TC. However, epigenetic alterations in the 3 genes, apart from GHSR, are not involved in the tumorigenesis of thymoma.

\section{Acknowledgements}

The authors would like to thank Dr. Yoshihiro Okayama, Clinical Trial Center For Developmental Therapeutics, Tokushima University Hospital for the statistical analysis.

\section{Funding}

No funding was received.

\section{Availability of data and materials}

The datasets used and/or analyzed during the present study are available from the corresponding author on reasonable request. 


\section{Authors' contributions}

RK, KKo, SS, MT and KKa analyzed and interpreted patient data regarding DNA methylation using pyrosequencing. YK, NK, TS, HTo and MY collected patient frozen materials for tumor and thymic tissue and patient clinical data. KKo, HTa and AT designed and carried out this project. KKo and HTa performed the histological examination of thymic epithelial tumors, and RK, KKo and AT were major contributors in the writing of the manuscript. All authors have read and approved the final manuscript.

\section{Ethics approval and consent to participate}

The present study was performed in accordance with the principles outlined in the Declaration of Helsinki. Following the approval of all aspects of this study by the local Ethics Committee (Tokushima University Hospital, approval numbers. 2205-4), formal written consent was obtained from all patients. In addition, formal written consent for the publication of any associated data was obtained from all patients.

\section{Patient consent for publication}

Not applicable.

\section{Competing interests}

The authors declare that they have no competing interests.

\section{References}

1. Kondo K: Therapy for thymic epithelial tumors. Gen Thorac Cardiovasc Surg 62: 468-474, 2014.

2. Travis WD, Brambilla E, Burke AP, Marx A and Nicholson AG: WHO classification of tumours of the lung, pleura, thymus and heart. In: World Health Organization classification of tumours. Bosman FT, Jaffe ES, Lakhani SR and Ohgaki H (eds). 4th edition. IARC Press, Lyon, pp183-243, 2015.

3. Shimosato Y, Mukai K and Matsuno Y: Tumors of the mediastinum. In: AFIP Atlas of tumor pathology, Series 4. Armed Forces Institute of Pathology, Washington, DC, 2010.

4. Wang Y, Thomas A, Lau C, Rajan A, Zhu Y, Killian JK, Petrini I, Pham T, Morrow B, Zhong X, et al: Mutations of epigenetic regulatory genes are common in thymic carcinomas. Sci Rep 4: 7336, 2014

5. Radovich M, Pickering CR, Felau I, Ha G, Zhang H, Jo H, Hoadley KA, Anur P, Zhang J, McLellan M, et al: The integrated genomic landscape of thymic epithelial tumors. Cancer Cell 33: 244-258.e10, 2018.

6. Petrini I, Meltzer PS, Kim IK, Lucchi M, Park KS, Fontanini G, Gao J, Zucali PA, Calabrese F, Favaretto A, et al: A specific missense mutation in GTF2I occurs at high frequency in thymic epithelial tumors. Nat Genet 46: 844-849, 2014.

7. Hirose Y, Kondo K, Takizawa H, Nagao T, Nakagawa Y, Fujino H, Taba H, Kenzaki K, Sakiyama S and Tangoku A: Aberrant methylation of tumour-related genes in thymic epithelial tumours. Lung Cancer 64: 155-159, 2009.

8. Mokhtar M, Kondo K, Namura T, Ali AH, Fujita Y, Takai C, Takizawa H, Nakagawa Y, Toba H, Kajiura K, et al: Methylation and expression profiles of MGMT gene in thymic epithelial tumors. Lung Cancer 83: 279-287, 2014.

9. Detterbeck FC, Nicholson AG, Kondo K, Van Schil P and Moran C: The Masaoka-Koga stage classification for thymic malignancies: Clarification and definition of terms. J Thorac Oncol 6 (7 Suppl 3): S1710-S1716, 2011.
10. Kajiura K, Masuda K, Naruto T, Kohmoto T, Watanabe M, Tsuboi M, Takizawa H, Kondo K, Tangoku A and Imoto I: Frequent silencing of the candidate tumor suppressor TRIM58 by promoter methylation in early-stage lung adenocarcinoma. Oncotarget 8: 2890-2905, 2017.

11. Moskalev EA, Jandaghi P, Fallah M, Manoochehri M, Botla SK, Kolychev OV, Nikitin EA, Bubnov VV, von Knebel Doeberitz M, Strobel O, et al: GHSR DNA hypermethylation is a common epigenetic alteration of high diagnostic value in a broad spectrum of cancers. Oncotarget 6: 4418-4427, 2015.

12. Pal J, Patil V, Mondal B, Shukla S, Hegde AS, Arivazhagan A, Santosh V and Somasundaram K: Epigenetically silenced GNG4 inhibits SDF1 $\alpha /$ CXCR4 signaling in mesenchymal glioblastoma. Genes Cancer 7: 136-147, 2016.

13. Marzese DM, Scolyer RA, Huynh JL, Huang SK, Hirose H, Chong KK, Kiyohara E, Wang J, Kawas NP, Donovan NC, et al: Epigenome-wide DNA methylation landscape of melanoma progression to brain metastasis reveals aberrations on homeobox D cluster associated with prognosis. Hum Mol Genet 23: 226-238, 2014.

14. Misawa K, Mochizuki D, Imai A, Misawa Y, Endo S, Mima M, Kawasaki H, Carey TE and Kanazawa T: Epigenetic silencing of SALL3 is an independent predictor of poor survival in head and neck cancer. Clin Epigenetics 9: 64, 2017.

15. Kojima M, Hosoda H, Date Y, Nakazato M, Matsuo H and Kangawa K: Ghrelin is a growth-hormone-releasing acylated peptide from stomach. Nature 402: 656-660, 1999.

16. Howard AD, Feighner SD, Cully DF, Arena JP, Liberator PA, Rosenblum CI, Hamelin M, Hreniuk DL, Palyha OC, Anderson J, et al: A receptor in pituitary and hypothalamus that functions in growth hormone release. Science 273: 974-977, 1996.

17. Lin TC and Hsiao M: Ghrelin and cancer progression. Biochim Biophys Acta Rev Cancer 1868: 51-57, 2017.

18. Jandaghi P, Hoheisel JD and Riazalhosseini Y: GHSR hypermethylation: A promising pan-cancer marker. Cell Cycle 14: 689-690, 2015.

19. Clapham DE and Neer EJ: G protein beta gamma subunits. Annu Rev Pharmacol Toxicol 37: 167-203, 1997.

20. Maina EN, Morris MR, Zatyka M, Raval RR, Banks RE, Richards FM, Johnson CM and Maher ER: Identification of novel VHL target genes and relationship to hypoxic response pathways. Oncogene 24: 4549-4558, 2005.

21. McGinnis W and Krumlauf R: Homeobox genes and axial patterning. Cell 68: 283-302, 1992.

22. Shah N and Sukumar S: The Hox genes and their roles in oncogenesis. Nat Rev Cancer 10: 361-371, 2010.

23. Tabuse M, Ohta S, Ohashi Y, Fukaya R, Misawa A, Yoshida K, Kawase T, Saya H, Thirant C, Chneiweiss H, et al: Functional analysis of HOXD9 in human gliomas and glioma cancer stem cells. Mol Cancer 10: 60, 2011.

24. Lv X, Li L, Lv L, Qu X, Jin S, Li K, Deng X, Cheng L, He H and Dong L: HOXD9 promotes epithelial-mesenchymal transition and cancer metastasis by ZEB1 regulation in hepatocellular carcinoma. J Exp Clin Cancer Res 34: 133, 2015.

25. Kohlhase J, Hausmann S, Stojmenovic G, Dixkens C, Bink K, Schulz-Schaeffer W, Altmann M and Engel W: SALL3, a new member of the human spalt-like gene family, maps to $18 \mathrm{q} 23$. Genomics 62: 216-222, 1999.

26. Strathdee G, Sutherland R, Jonsson JJ, Sataloff R, Kohonen-Corish M, Grady D and Overhauser J: Molecular characterization of patients with 18q23 deletions. Am J Hum Genet 60: 860-868, 1997.

27. Yang XX, Sun JZ, Li FX, Wu YS, Du HY, Zhu W, Li XH and $\mathrm{Li} \mathrm{M}$ : Aberrant methylation and downregulation of sall3 in human hepatocellular carcinoma. World J Gastroenterol 18: 2719-2726, 2012.

28. Shikauchi Y, Saiura A, Kubo T, Niwa Y, Yamamoto J, Murase Y and Yoshikawa H: SALL3 interacts with DNMT3A and shows the ability to inhibit $\mathrm{CpG}$ island methylation in hepatocellular carcinoma. Mol Cell Biol 29: 1944-1958, 2009.

29. Wei X, Zhang S, Cao D, Zhao M, Zhang Q, Zhao J, Yang T, Pei M, Wang L, Li Y and Yang X: Aberrant hypermethylation of SALL3 with HPV involvement contributes to the carcinogenesis of cervical cancer. PLoS One 10: e0145700, 2015. 